\title{
MAXIMAL FUCHSIAN GROUPS
}

\author{
BY LEON GREENBERG ${ }^{1}$
}

Communicated by Lipman Bers, February 25, 1963

1. Definitions. Let $D$ be the unit disk $\{z|| z \mid<1\}$ and let $\mathscr{L}$ be the group of conformal homeomorphisms of $D$. A Fuchsian group is a discrete subgroup of $\&$. We shall be concerned here with the finitely generated Fuchsian groups. It is known that these have the following presentations.

Generators: $a_{1}, b_{1}, \cdots, a_{g}, b_{g}, e_{1}, \cdots, e_{k}, h_{1}, \cdots, h_{m}, p_{1}, \cdots, p_{r}$.

Defining relations: $e_{1}^{\nu_{1}}=e_{2}^{\nu_{2}}=\cdots=e_{k}^{\nu_{k}}=1$,

$$
\left(\prod_{i=1}^{o} a_{i} b_{i} a_{i}^{-1} b^{-1}\right) e_{1} \cdots e_{k} h_{1} \cdots h_{m} p_{1} \cdots p_{n}=1 .
$$

A group of the above type will be denoted $F\left(g ; \nu_{1}, \cdots, \nu_{k} ; m ; n\right)$. The elements $h_{i}$ and $p_{j}$ are not distinguishable in the abstract group $F$, but depend on the imbedding of $F$ in $\&$. The elements $h_{i}$ are hyperbolic, $p_{j}$ are parabolic, and these correspond respectively to the boundary curves and punctures in the Riemann surface $D / F$. These elements $h_{i}, p_{j}$ and their conjugates in $F$ will be called the boundary elements of $F$.

We shall say that a finitely generated Fuchsian group $F$ is finitely maximal ( $f$-maximal) if there does not exist any other Fuchsian group $G$ such that $F \subset G$ and the index $[G: F]$ is finite. We note that if $F$ does not have any hyperbolic boundary elements, then $F$ is $f$-maximal if and only if there does not exist any other Fuchsian group which contains it. On the other hand, if $F$ does have hyperbolic boundary elements, then there always exist Fuchsian groups $G$ which contain $F$ with infinite index.

By a geometric isomorphism (g-isomorphism) of a Fuchsian group $F$, we shall mean an isomorphism $\gamma: F \rightarrow \mathscr{L}$, such that

(1) $\gamma(F)$ is a Fuchsian group.

(2) $\gamma$ maps the hyperbolic (parabolic) boundary elements of $F$ onto the hyperbolic (parabolic) boundary elements of $\gamma(F)$. Let $\Gamma(F)$ denote the set of $g$-isomorphisms of $F . \Gamma(F)$ can be topologized in the following way. Let $f_{1}, \cdots, f_{n}$ be a set of generators for $F . \Gamma(F)$ can be imbedded in $\mathscr{2}^{n}$ by assigning to $\gamma \in \Gamma(F)$ the point $\left(\gamma\left(f_{1}\right), \cdots, \gamma\left(f_{n}\right)\right) \in \mathcal{L}^{n} . \Gamma(F)$ is given the relative topology in $\mathfrak{L}^{n}$. We introduce an equivalence relation $\rho$ in $\Gamma(F)$. Let $\mathcal{L}^{\prime}$ denote the

${ }^{1}$ This work has been supported by the Office of Naval Research. 
group of angle-preserving homeomorphisms of $D$. ( $\mathcal{L}^{\prime}$ contains orientation reversing transformations. $\mathscr{L}$ is a subgroup of index 2 in $\mathfrak{L}^{\prime}$.) If $\gamma_{1}, \gamma_{2} \in \Gamma(F)$, say that $\gamma_{1}$ is $\rho$-equivalent to $\gamma_{2}$ if there exists $\lambda \in \mathscr{L}^{\prime}$ such that

$$
\gamma_{2}(f)=\lambda \gamma_{1}(f) \lambda^{-1}
$$

for all elements $f \in F$. The quotient space

$$
T(F)=\Gamma(F) / \rho
$$

is then the analogue for $F$ of the Teichmüller space of a Riemann surface. $T(F)$ has been investigated in recent work by L. Ahlfors $[1 ; 2]$, L. Bers $[3 ; 4 ; 5]$, W. Fenchel and J. Nielsen [6] (and further unpublished work by the first two authors). Among other things, it is known that if $F$ is finitely generated, then $T(F)$ is a finite dimensional cell. We shall use the Fenchel-Nielsen theory to establish the results announced in this note.

Let $A(F)$ be the group of $g$-automorphisms of a Fuchsian group $F$, and let $I(F)$ be the subgroup of inner automorphisms. The modular group $M(F)$ is defined as the quotient group:

$$
M(F)=A(F) / I(F) .
$$

$M(F)$ operates in $T(F)$ in the following way. Let $\alpha \in A(F)$, and consider the map $\Gamma(F) \rightarrow \Gamma(F)$ defined by $\gamma \rightarrow \gamma \circ \alpha$. As $\alpha$ varies in its $I(F)$-coset, $\gamma \circ \alpha$ varies in its $\rho$-equivalence class. Therefore, this induces an operation of $M(F)$ on $T(F)$. It is known that $M(F)$ is a properly discontinuous group of transformations of $T(F)$, when $F$ is finitely generated.

\section{The results.}

TheOREM $1 .{ }^{2}$ Let $F$ and $G$ be finitely generated Fuchsian groups such that $F \subset G$ and the index $[G: F]$ is finite. Let $\iota: F \rightarrow G$ denote the injection map. The map $\Gamma(G) \rightarrow \Gamma(F)$, defined by $\gamma \rightarrow \gamma \circ$ เ induces a map

$$
m: T(G) \rightarrow T(F)
$$

which has the following properties.

(1) $m$ is real analytic and $1-1$.

(2) The image $I=m[T(G)]$ is a closed subset of $T(F)$.

(3) The images of I under the modular group $M(F)$ do not accumulate in $T(F)$.

2 The author has been informed that statements (1) and (2) of Theorem 1 are contained in the Ahlfors-Bers theory. 
Let $\operatorname{Max}(F)$ denote the set of points in $T(F)$ which represent $f$ maximal groups. Theorem 1 together with certain area considerations lead to the following.

THEOREM 2. Let $F$ be a finitely generated Fuchsian group. Then one of the following is true.

(1) $\operatorname{Max}(F)$ is empty. There is a group $G$ which contains $F$ with finite index, such that $m[T(G)]=T(F)$ (where $m$ is the map in Theorem 1).

(2) $\operatorname{Max}(F)$ is an open, everywhere dense subset of $T(F)$, whose complement is an analytic set.

Thus, $\operatorname{Max}(F)$ is either the empty set, or it is most of $T(F)$. By some computations which utilize the Fenchel-Nielsen area and modulus formulas, we can actually find all groups $F$, such that $\operatorname{Max}(F)$ is empty.

THEOREM 3A. The following groups $F$ are the only finitely generated Fuchsian groups for which $\operatorname{Max}(F)$ is empty.

(a) Groups whose limit set consists of two points or less (i.e., cyclic groups and the group $F(0 ; 2,2 ; 1 ; 0))$.

(b) Certain triangle groups (which will be enumerated below).

(c) The groups $F$ in the following list. Next to each group $F$, we have listed the unique group $G$, such that $F$ is a subgroup of finite index in $G$, and $m[T(G)]=T(F)$. The index $[G: F]$ is always 2 .

\begin{tabular}{l|l}
\multicolumn{1}{c|}{$F$} & \multicolumn{1}{|c}{$G$} \\
\hline 1. $F(0 ;-; 1 ; 2)$ & $F(0 ; 2 ; 1 ; 0)$ \\
2. $F(0 ; n, n ; 1 ; 0)$ & $F(0 ; 2, n ; 1 ; 0)$ \\
3. $F(1 ;-; 1 ; 0)$ & $F(0 ; 2,2,2 ; 1 ; 0)$ \\
4. $F(0 ;-; 0 ; 4)$ & $F(0 ; 2,2 ; 0 ; 2)$ \\
5. $F(1 ;-; 0 ; 2)$ & $F(0 ; 2,2,2,2 ; 0 ; 1)$ \\
6. $F(0 ; m, m, n, n ; 0 ; 0)$ & $F(0 ; 2,2, m, n ; 0 ; 0)$ \\
7. $F(1 ; 2,2 ; 0 ; 0)$ & $F(0 ; 2,2,2,2,2 ; 0 ; 0)$ \\
8. $F(2 ;-; 0 ; 0)$ & $F(0 ; 2,2,2,2,2,2 ; 0 ; 0)$
\end{tabular}

In case 2 of the above list, $n>2$, and in case $6,1 / m+1 / n<1$. In cases $4-5, D / F$ has finite area, and in cases $6-8, D / F$ is compact. One would expect case 8 to appear, since every surface of genus 2 is hyperelliptic.

We shall denote the triangle group $F(0 ; a, b, c ; 0 ; 0)$ by $T(a, b, c)$, 
and the parabolic triangle groups $F(0 ; a, b ; 0 ; 1), F(0 ; a ; 0 ; 2)$ and $F(0 ;-; 0 ; 3)$ by $T(a, b, \infty), T(a, \infty, \infty)$ and $T(\infty, \infty, \infty)$, respectively. If $F$ is a triangle group, then $F$ has no deformations, so $T(F)$ consists of a single point. On the other hand, if $T(F)$ consists of a single point, then $F$ is either a triangle group, or an elliptic or parabolic cyclic group. It follows from Theorem 1 that the only groups which can possibly contain a triangle group are other triangle groups.

THEOREM 3B. The following groups $F$ are the only triangle groups for which $\operatorname{Max}(F)$ is empty (i.e., these are the only triangle groups which are not $f$-maximal).

(1) $F=T(m, m, n)$ is contained in $G=T(2, m, 2 n)$ with index 2.

(2) $F=T(2, n, 2 n)$ is contained in $G=T(2,3,2 n)$ with index 3.

(3) $F=T(3, n, 3 n)$ is contained in $G=T(2,3,3 n)$ with index 4.

In the above theorem, $m$ and $n$ are allowed to take on the value $\infty$. We remark that the above inclusion relations are not all that occur between triangle groups. There are also the following relations:

(4) $T(\infty, \infty, \infty) \subset T(3,3, \infty)$ with index 3 .

(5) $T(\infty, \infty, \infty) \subset T(2,4, \infty)$ with index 4 .

(6) $T(4,4,5) \subset T(2,4,5)$ with index 6 .

(7) $T(7,7,7) \subset T(2,3,7)$ with index 24 .

The above relations $1-7$ and those that follow from them are all inclusion relations between triangle groups. We also note that the modular group $T(2,3, \infty)$ has not been listed in Theorem $3 \mathrm{~B}$, and so it is $f$-maximal.

Let $F$ be a Fuchsian group of type $F(g ;-; 0 ; 0)$ (i.e., a group which uniformizes a Riemann surface of genus $g$ ). Let $S$ be the Riemann surface $D / F$. If $N$ is the normalizer of $F$ in $\mathscr{L}$, then the conformal group $C(S)$ is isomorphic to $N / F$. The previous results imply that for $g>2$, most of the groups isomorphic to $F$ are $f$-maximal. Thus $N=F$ and $C(S)=\{1\}$ for most Riemann surfaces of genus $g>2$. The following related result can be proved by constructing homomorphisms of Fuchsian groups onto finite groups.

THEOREM 4. Let $G$ be a nontrivial, finite group. Then there exists a closed Riemann surface $S$ whose conformal group $C(S)$ is isomorphic to $G$. $S$ may be chosen so that the quotient surface $T=S / C(S)$ has any preassigned genus.

Let $\mathscr{F}(S)$ and $\mathscr{F}(T)$ be the fields of meromorphic function on $S$ and $T$, respectively. Then $\mathcal{F}(S)$ is a Galois extension of $\mathcal{F}(T)$, whose Galois group is isomorphic to $G$. In particular, if we choose $T$ to be the 
sphere, then we have found a Galois extension of the field of rational functions, such that the Galois group is isomorphic to the preassigned group $G$.

\title{
REFERENCES
}

1. L. Ahlfors, The complex analytic structure of the space of closed Riemann surfaces, Analytic functions, pp. 45-66. Princeton Univ. Press, Princeton, N. J., 1960.

2. - Teichmiiller spaces, Proceedings of the International Congress of Mathematicians, 1962 (to appear).

3. L. Bers, Quasiconformal mappings and Teichmüller's theorem, Analytic functions, pp. 89-119, Princeton Univ. Press, Princeton, N. J., 1960.

4. - Spaces of Riemann surfaces, Proceedings of the International Congress of Mathematicians, 1958, pp. 349-361, Cambridge Univ. Press, New York, 1960.

5. - Correction to "Spaces of Riemann surfaces as bounded domains," Bull. Amer. Math. Soc. 67 (1961), 465-466.

6. W. Fenchel and J. Nielsen, Discontinuous groups of non-Euclidean motions (to appear).

BROWN UNIVERSITY

\section{A NOTE ON ENTIRE FUNCTIONS AND A CON JECTURE OF ERDÖS}

\author{
BY ALFRED GRAY AND S. M. SHAH ${ }^{1}$
}

Communicated by R. C. Buck, March 14, 1963

1. Introduction. Let $f(z)=\sum_{0}^{\infty} a_{n} z^{n}$ be an entire (transcendental) function and let

$$
M(r)=M(r, f)=\max _{|z|=r}|f(z)|, \quad \mu(r)=\mu(r, f)=\max _{n}\left(\left|a_{n}\right| r^{n}\right) .
$$

Erdös conjectured that [1] for every entire function, either

$$
U=U(f) \equiv \limsup _{r \rightarrow \infty} \mu(r) / M(r)>u=u(f) \equiv \liminf _{r \rightarrow \infty} \mu(r) / M(r),
$$

or

$$
U(f)=0 .
$$

We prove this conjecture, except in one case, when broadly speaking the Taylor series for $f(z)$ has "wide latent" gaps. For $r>0$, let $\nu(r)$ $=\max \left(n|\mu(r)=| a_{n} \mid r^{n}\right)$, and denote by $\left\{\rho_{n}\right\}$ the sequence of jump-

1 The work of this author was supported by U. S. National Science Foundation grant N.S.F. GP-209. 